\title{
$\mathfrak{D a n n s} \mathfrak{W e e r e n}$
}

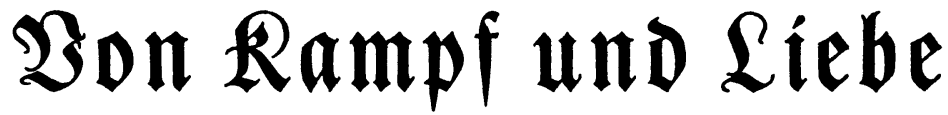

Lieder a us meiner

Gammelmappe, aud

zur $\mathfrak{L} \mathfrak{a} u \mathfrak{t e}$ zu fingen

Springer fachmedien Wiesbaden Gmb6 
Oämtlibe Lieder diefes beftes find Originallompofitionen von Sanns beeren und damit urbeberredtlid gef hübt. Radbrud if nur mit Genebmigung des Berlages geftattet.

ISBN 978-3-322-98373-2 ISBN 978-3-322-99117-1 (eBook)

DOI 10.1007/978-3-322-99117-1

Titelzeidnung von $\mathfrak{X}$ udi $\mathfrak{b}$ oppe

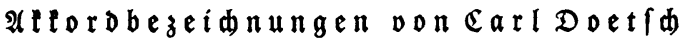




\section{Eín Bort voraus:}

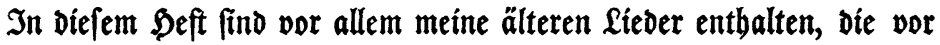
Jabren ime einen oder anderen Liederbeft gedrultt wurben, bas nun Iange vergriffen ît. Da war es mir ein lieber Gedante, einmal eíne größere $\mathfrak{A} \mathfrak{n} z \mathfrak{b l} l$ meiner Lièder uménander zu verfammeln.

Einige von ibnen erwarben fid fthon vor dem $\mathfrak{B e l t t r i e g e ~ e i n e n ~ g r o b e n ~}$ Greundestreis, mit Den im Rriege entftandenen aber tonnte id meinen Rameraben mande Entfpannung und Greube bringen, begleitete mich Dod meine Laute fhon feit $\mathfrak{A n f a n g} 1915$ in bie Odü̈bengräben wie ppäter zu den Fliegern.

Randes der vorliegenden Lieder entftand vorn am Seinde, im Unter, ftand oder im Xubequartier gleiđ hinter Den Gräben. Einige der $\mathfrak{B e i f e n ~}$ find zu meiner Greude in Das beute lebendige Liedgut Deutf(tlands eingegangen.

Reben Diefen Liedern fteben bier aber aud einige wenige bisber uns georutt gebliebene $\mathfrak{B e i f e n , ~ b i e ~ n u n ~ z u m ~ e r f t e n ~ M a l e ~ b i n a u s g e b e n , ~ f i ́ ~}$ Greunde zu werben. Selingt ihnen Das, Dann foll bald eín neues beft aus meiner Ĺédermappe folgen.

Alle Lieder wurden mit einer Attordbezeidnung verfeben, die die Bes, gleitung auf Der Laute Dder bem 2 Atlordeon obne weiteres jedem ermögs líbt, Der nur einige mufitalif he Renntniffe bat. Der grobe $\mathfrak{B} u d$ ftabe bezeidnet Den Durs, Der Hleine Den Midldreitlang; Die Llmelebrungen find Durd Den Darunterge ebten $\mathfrak{B a ß t o n}$ (in Ileinen Budftaben) gelenn zeidnnet. Eine zugefügte 7 verlangt den Geptimenaltord. Eine Null mit Darunterftebender Tonbezeidnung in tleinen 3 udfftaben gibt an, Daß nur mit biefem $\mathfrak{B a} \mathfrak{B}$ ton begleitet wird.

Siogge aud dies tleine $\mathfrak{p e f t}$ wie all meine früheren feine Greunde finden.

Serf ord, im Grübjabr 1938

Sanns Seeren 